\title{
Estimation of Optimal Values for Lumped Elements in a Finite Element - Lumped Parameter Model of a Loudspeaker
}

Nielsen, Daniel Gert; Andersen, Peter Risby; Jensen, Jakob Søndergaard; Agerkvist, Finn T.

Published in:

Journal of Computational Acoustics

Link to article, DOI:

$10.1142 / S 2591728520500127$

Publication date:

2020

Document Version

Peer reviewed version

Link back to DTU Orbit

Citation (APA):

Nielsen, D. G., Andersen, P. R., Jensen, J. S., \& Agerkvist, F. T. (2020). Estimation of Optimal Values for Lumped Elements in a Finite Element - Lumped Parameter Model of a Loudspeaker. Journal of Computational Acoustics, 28(2), [2050012]. https://doi.org/10.1142/S2591728520500127

\section{General rights}

Copyright and moral rights for the publications made accessible in the public portal are retained by the authors and/or other copyright owners and it is a condition of accessing publications that users recognise and abide by the legal requirements associated with these rights.

- Users may download and print one copy of any publication from the public portal for the purpose of private study or research.

- You may not further distribute the material or use it for any profit-making activity or commercial gain

- You may freely distribute the URL identifying the publication in the public portal 


\title{
Estimation of Optimal Values for Lumped Elements in a Finite Element - Lumped Parameter Model of a Loudspeaker
}

\author{
Daniel Gert Nielsen, Peter Risby Andersen, Jakob Søndergaard Jensen, Finn Thomas Agerkvist
}

December 14, 2020

\begin{abstract}
Finite element methods are progressively being utilized to assist in the continuous development of loudspeakers. The core of this paper is the method of lumping certain parts of the finite element model, creating a significant reduction in the model complexity that allows for e.g. faster structural optimization. This is illustrated in the paper with a loudspeaker example where the electromagnetic parts are lumped as well as the spider. It is shown that the simplified model still matches the complex response of the full FE model at very high frequencies.
\end{abstract}

\section{Introduction}

Loudspeakers have been an essential part of society for over a century, the performance requirements vary depending on the situation and environment in which said loudspeaker is operating in. Therefore, a great effort is made in order to analyse and predict the behaviour of loudspeakers. Simulating a loudspeaker in a numerical model is a complex task with respect to modelling the physics in a finite element (FE) model and the associated long computational times. When considering a particular aspect of the loudspeaker performance, one may simplify and lump those parts of the speaker that have little influence in the aspect studied, for the linear response at high frequencies, the electromagnetic part may be lumped as the diaphragm and surround are the most substantial parts. This approach can be beneficial in many cases, for instance when optimizing mechanical structures where many calculations are carried out repeatedly, in-order to find an optimized design. Optimization of acoustic-structure problems is a rapidly growing research field, examples of the applicability can be seen in Refs. $[1,2,3,4,5]$ and in Refs. [6, 7] examples of loudspeaker optimization can be found. Therefore, we propose a numerical method for predicting the behaviour of a loudspeaker in its linear operating range in a simple and efficient manner. The proposed method combines two well known techniques, namely the FE method [8] and a lumped parameter model (LPM) [9].

The combination of the FE method and LPM is made such that structural optimization ultimately can be performed on the diaphragm of the loudspeaker, therefore, this part must be considered with a FE-model. A LPM is then utilized to model the entire electric motor system together with the voice-coil (VC), VC former and the spider in order to reduce the complexity of the modelling task and the calculation time.

Utilizing a combination of FE and Lumped Elements (LE) is not novel. It is especially common within the research topics regarding balanced-armature receivers and MEMS microphones. Recently Sun et. al. presented a modelling technique where a FE-LE model was used to model a simplified balanced-armature receiver [10]. Schrag et. al. enhances the design of microsystems by combining a lumped model with a distributed system level model [11]. A computational example in which a balanced-armature receiver itself is represented by a LPM that is then coupled to a FE model of the vibration isolation system is shown in Ref. [12]. Marttila and Jensen [13] briefly presents an approach for incorporating a LPM in conjunction with a FE-model.

If the amplitude of the movement of the speaker is small, the loudspeaker is in its linear operating range, which simplifies the required measurement technique. The small-signal parameters can be measured dynamically in conjunction with a system identification technique such as the Klippel system described in Ref. [14]. This measurement technique will yield the Thiele/Small parameters which can be used in a LPM of the loudspeaker. Klippel describes a measuring technique using a laser scanner to obtain the distributed mechanical parameters 
which could be used in a FE model of e.g. the loudspeaker cone[15]. Furthermore, Cardenas and Klippel estimated an effective frequency dependent and complex Young's modulus by fitting FE simulations to measurements of a loudspeaker [16].

The method disclosed in this paper can be used to fit a numerical model to measurements of a loudspeaker. Ideally the measurements would contain values of the impedance as a function of frequency and/or measurements of the magnitude and phase of the pressure 1 meter away from the unit. The measured data is then used to optimally configure the parameters in the LPM, such that the impedance and radiated sound from the loudspeaker unit in the numerical model resemble the authentic unit.

This paper is a continuation of the work presented in Ref. [17], but here we study the geometry of an actual 5 inch loudspeaker instead of a flat panel loudspeaker. The lumped circuit model is extended to include eddy currents by considering the inductor with a fractional order derivative model. Furthermore, an optimization algorithm has been implemented, which can fit the lumped components in the proposed numerical model to represent an actual loudspeaker either with parameters from a data sheet or actual measurements.

The paper is structured such that the theory required to combine the FE method with the LPM is presented together with the optimization problem used for estimating the optimal lumped parameters. Succeeding sections will contain relevant numerical results and comparisons together with a discussion and conclusion about the results.

\section{Theory}

Figure 1 shows a sketch of the model problem, which is a loudspeaker mounted in a baffle. The dashed box in the figure show the components that are contained in the LPM. Namely, the entire electric motor system, the VC, VC former and the spider. The rest of the geometry and the surrounding unbounded acoustic domain are modelled with a FE-model. The two models are linked together in "coupling node $i$ ", referring to a specific node in the FE-model. This section serves to establish a system of equations that can readily be used to solve an unbounded acousticmechanical interaction problem, then couple it to a LPM model, in which the values of the lumped components are estimated based on measurements.

\section{$2.1 \quad$ Finite element model}

The numerical model is used to simulate the acoustic wave propagation in an unbounded domain caused by a moving coil loudspeaker. The modelling setup can be seen in Fig. 2, note that the loudspeaker geometry is exaggerated for explanatory purposes. The loudspeaker geometry and acoustic domain is studied by utilizing an axisymmetric FE model, which implies that the geometry in Fig. 2 is revolved around the z-axis. Perfectly Matched Layers (PMLs) have been used to truncate the unbounded domain into a finite domain [18].

The governing equation for the time-harmonic motion of a linear elastic body where body forces has been neglected can be written as

$$
\begin{aligned}
& -\rho \omega^{2} \mathbf{u}-\nabla \cdot \boldsymbol{\sigma}(\mathbf{u})=0 \quad \text { in } \Omega_{s} \\
& \boldsymbol{\sigma}=\mathbf{C} \boldsymbol{\epsilon} \\
& \epsilon=\left\{\epsilon_{r} \epsilon_{\theta} \epsilon_{z} \gamma_{z r}\right\}^{T} \quad \text { (2D Axisymmetric) } \\
& \epsilon_{r}=\frac{\partial u_{r}}{\partial r}, \epsilon_{\theta}=\frac{u_{r}}{r}, \epsilon_{z}=\frac{\partial u_{z}}{\partial z}, \gamma_{z r}=\left(\frac{\partial u_{r}}{\partial z}+\frac{\partial u_{z}}{\partial r}\right)
\end{aligned}
$$

here $\rho$ is the mass density of the material, $\mathbf{u}$ is the structural displacements, $\boldsymbol{\sigma}$ is the stress tensor, $\omega$ is the excitation frequency in radians, $\Omega_{s}$ is the structural domain $\mathbf{C}$ is the constitutive matrix for an axisymmetric structure, $\boldsymbol{\epsilon}$ is the strain tensor, $u_{r}$ is the structural displacement in the r-direction and $u_{z}$ is the displacement in the z-direction. Applying the Galerkin method to Eq. (2) and transformation into the frequency domain, yields the following FE equation

$$
\left(\mathbf{K}-\omega^{2} \mathbf{M}\right) \mathbf{u}=\mathbf{f},
$$

where the capital bold letters specifies a matrix, small bold letters implies a vector, non bold letters are scalars. The externally applied time harmonic force is denoted $\mathbf{f}, \mathbf{K}$ is the structural stiffness matrix and $\mathbf{M}$ is the structural 


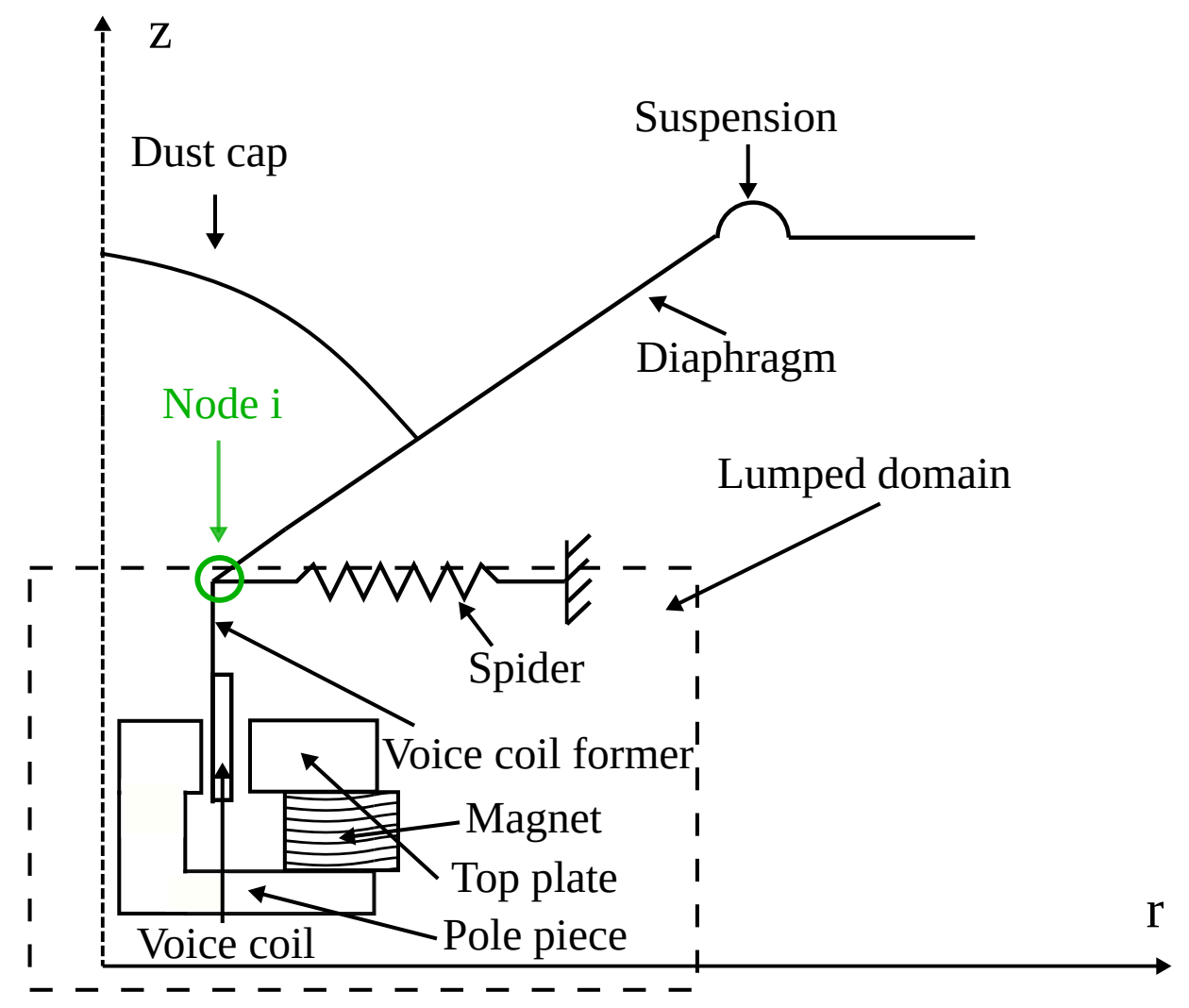

Figure 1: 2D sketch of a loudspeaker placed in an infinite baffle 


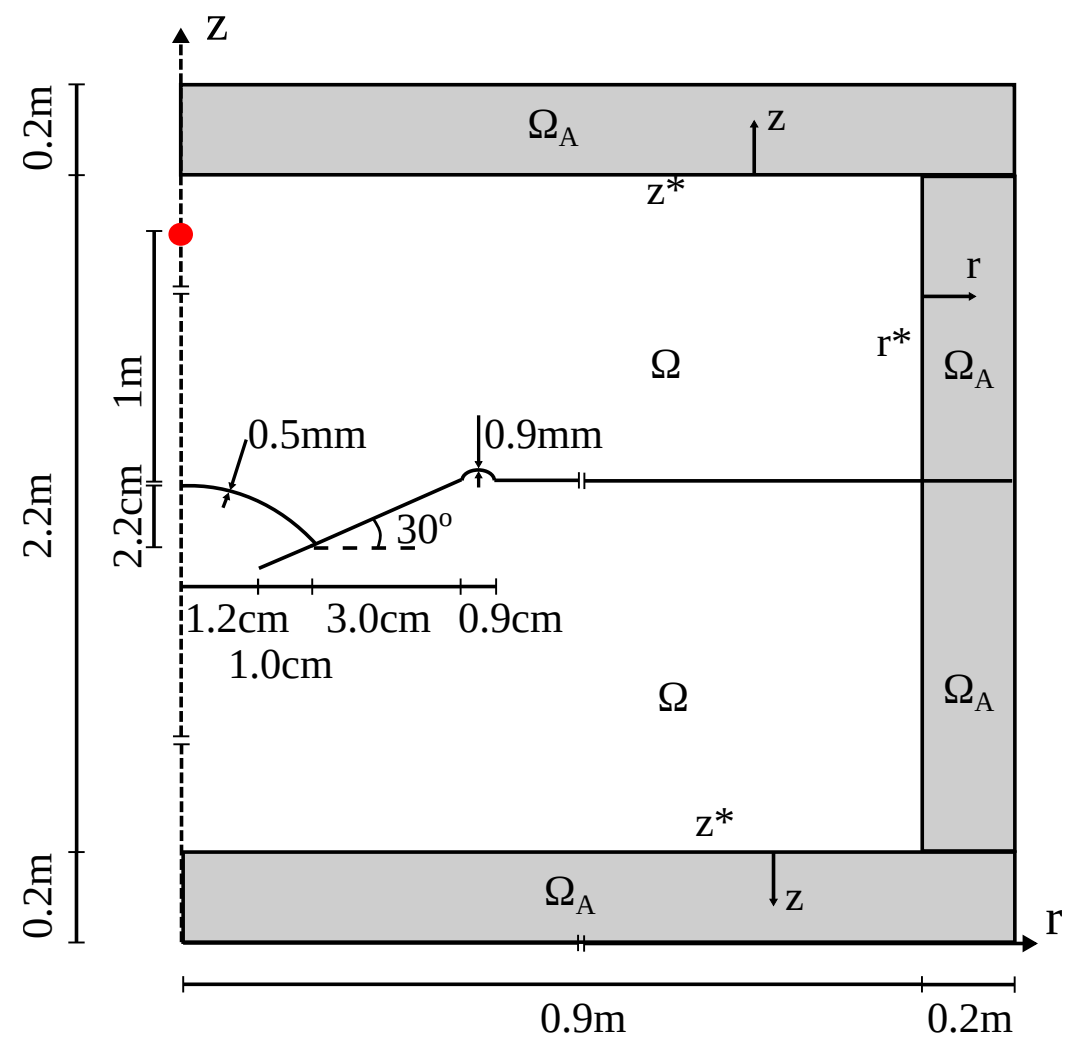

Figure 2: Sketch of the loudspeaker geometry, PML regions, the acoustic domain and the location of the measuring point for the pressure.

mass matrix which can be written as

$$
\mathbf{K}=\int_{\Omega_{s}} \int_{-\pi}^{\pi} \mathbf{B}^{T} \mathbf{C B} r \mathrm{~d} \Omega_{s}, \mathbf{M}=\int_{\Omega_{s}} \int_{-\pi}^{\pi} \rho \mathbf{N}^{T} \mathbf{N} r \mathrm{~d} \Omega_{s}
$$

Here $\mathbf{B}$ is the strain-displacement matrix, $r$ is the radial distance to the Gauss point and $\mathbf{N}$ is a matrix consisting of the quadratic iso-parametric shape functions.

In this paper damping is considered as an isotropic structural loss factor, denoted $\eta$, such that $\mathbf{K}=\mathbf{K}(1+j \eta)$, where $j$ is the imaginary number, $\eta$ equals 0.25 in the rubber surround of the speaker and 0.2 in the diaphragm and dust cap.

In the acoustic domain the Helmholtz equation is solved in the frequency domain

$$
\Delta p+\frac{\omega^{2}}{c^{2}} p=0
$$

here $\Delta$ is the Laplace operator in cylindrical coordinates, $p$ is the pressure and $c$ is the speed of sound in air.

A modified Helmholtz equation[19, 20] is solved in a truncated PML region $\left(\Omega_{A}\right)$, as shown in Fig. 2.

$$
\frac{1}{\gamma_{r}} \frac{\partial}{\partial r}\left(\frac{1}{\gamma_{r}} \frac{\partial p_{A}}{\partial r}\right)+\frac{1}{\gamma_{z}} \frac{\partial}{\partial z}\left(\frac{1}{\gamma_{z}} \frac{\partial p_{A}}{\partial z}\right)+k^{2} p_{A}=0
$$

where $p_{A}$ is the pressure in the PML region, the formulation of $\gamma$ is from [21], here extended to accommodate for a 
PML in both the $r$ - and $z$ direction

$$
\begin{aligned}
& \gamma_{r}(r)=1-j \alpha\left(\frac{r-r^{*}}{t}\right)^{2} \\
& \gamma_{z}(z)=1-j \alpha\left(\frac{z-z^{*}}{t}\right)^{2} .
\end{aligned}
$$

Where $r^{*}$ and $z^{*}$, indicate the interface coordinate between the PML/acoustic domain and $r$ and $z$ are the position within the PML as shown in Fig. 2. $\alpha$ is the absorption coefficient with a constant value of 10 and $t$ is half the thickness of the PML which equates to $0.1 \mathrm{~m}$.

Equations (7) and (8) are written in the usual FE equations in the frequency domain

$$
\left(\mathbf{K}_{a}-\omega^{2} \mathbf{M}_{a}\right) \mathbf{p}=0 .
$$

Here $\mathbf{p}$ is the solution vector containing the nodal pressures, $\mathbf{M}_{a}$ is the acoustic mass matrix and $\mathbf{K}_{a}$ is the acoustic stiffness matrix which can be computed as

$$
\begin{aligned}
\mathbf{K}_{a} & =\int_{\Omega} \int_{-\pi}^{\pi}\left(\mathbf{N}_{a, r}^{T} \mathbf{N}_{a, r}+\mathbf{N}_{a, z}^{T} \mathbf{N}_{a, z}\right) r \mathrm{~d} \Omega+\int_{\Omega_{A}} \int_{-\pi}^{\pi}\left(\frac{\gamma_{z}}{\gamma_{r}} \mathbf{N}_{a, r}^{T} \mathbf{N}_{a, r}+\frac{\gamma_{r}}{\gamma_{z}} \mathbf{N}_{a, z}^{T} \mathbf{N}_{a, z}\right) r \mathrm{~d} \Omega_{A} \\
\mathbf{M}_{a} & =\int_{\Omega} \int_{-\pi}^{\pi} \frac{1}{c^{2}} \mathbf{N}_{a}^{T} \mathbf{N}_{a} r \mathrm{~d} \Omega+\int_{\Omega_{A}} \int_{-\pi}^{\pi} \frac{1}{c^{2}} \gamma_{r} \gamma_{z} \mathbf{N}_{a}^{T} \mathbf{N}_{a} r \mathrm{~d} \Omega_{A},
\end{aligned}
$$

where subscript $\mathrm{r}$ and $\mathrm{z}$ refers, respectively, to the differential operator with respect to global $\mathrm{r}$ - and z-coordinates, $\mathbf{N}_{a}$ is a row vector consisting of the quadratic acoustic shape functions, $\Omega$ and $\Omega_{A}$ refers to the acoustic domain and the PML, respectively.

The acoustic and mechanical domains are fully coupled at the shared interfaces such that when the mechanical structure vibrates it acts as an acoustic source, furthermore the surface pressure acting on the mechanical structure is accounted for. This is included in the coupling matrix $\mathbf{S}$

$$
\mathbf{S}=\int_{\Gamma} \int_{-\pi}^{\pi} \mathbf{N}^{T} \mathbf{n}_{a} \mathbf{N}_{a} r \mathrm{~d} \Gamma,
$$

where $\mathbf{n}_{a}$ is the normal vector of the interface between the acoustic and structural boundary pointing outwards from the acoustic boundary and $\Gamma$ refers to the interface between the acoustic and structural domain.

Combining Eq. 5 and 11 yields the entire system of equations for the FE-model[8]

$$
\left(\left[\begin{array}{cc}
\mathbf{K} & -\mathbf{S}^{T} \\
\mathbf{0} & \mathbf{K}_{a}
\end{array}\right]-\omega^{2}\left[\begin{array}{cc}
\mathbf{M} & \mathbf{0} \\
\rho \mathbf{S} & \mathbf{M}_{a}
\end{array}\right]\right)\left\{\begin{array}{l}
\mathbf{u} \\
\mathbf{p}
\end{array}\right\}=\left\{\begin{array}{l}
\mathbf{f} \\
\mathbf{0}
\end{array}\right\} .
$$

For the remainder of this paper Eq. (15) is written in a compact format

$$
\left(\tilde{\mathbf{K}}-\omega^{2} \tilde{\mathbf{M}}\right)\left\{\begin{array}{l}
\mathbf{u} \\
\mathbf{p}
\end{array}\right\}=\left\{\begin{array}{l}
\mathbf{f} \\
\mathbf{0}
\end{array}\right\} .
$$

\subsection{Coupled system}

Figure 3 is a schematic drawing of the lumped circuit model representing the entire electric motor system and the $\mathrm{VC}, \mathrm{VC}$ former and spider of the mechanical system. Information is passed between the LPM and the FE model in the coupling node $i$ located as shown on Fig. 1. The left circuit in Fig. 3 is a representation of the electrical motor system, where $e_{g}$ is the applied voltage from an AC source, $i_{c}$ is the current, $R_{E}$ is the DC resistance in the wire of the $\mathrm{VC}, L_{E}$ is the inductance of the $\mathrm{VC}$ and $e_{c}$ is the back-induced voltage caused by the movement of the loudspeaker. In the expression describing $e_{c}, B l$ is the force factor and $\dot{u}$ is the velocity of the movement.

The right circuit on Fig. 3 represents the material properties of the partly lumped mechanical components, the force applied from the electric motor system is $F^{e l e k}, M_{p, l u m p}$ is the total mass of the lumped components, $R_{p, l u m p}$ 

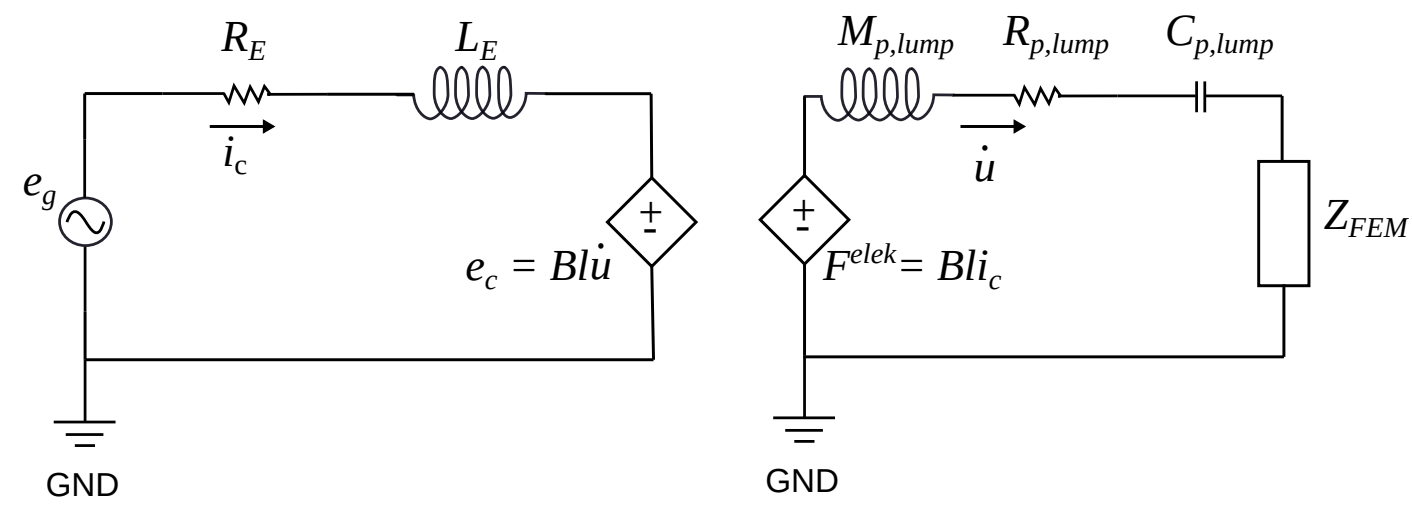

Figure 3: Lumped circuit model with partly lumped mechanical components

is the damping coefficient, $C_{p, l u m p}$ is the compliance and the impedance block, $Z_{F E M}$, represents the contribution from the FE model.

The equation for the electric motor system is represented in the frequency domain, in which the eddy currents in the inductor are included with a fractional order derivative model $[22,23]$

$$
e_{g}=R_{E} i_{c}+(j \omega)^{n} L_{E} i_{c}+B l \dot{u}_{i},
$$

where $n$ is the fractional order. The scalar equation for the mechanical components in Fig. 3 including the contribution from the FE-model via the impedance $Z_{F E M}=\frac{F_{i}}{j \omega \dot{u}_{i}}$, where sub-index $i$ refers to the value in the coupling node $i$ is

$$
B l i_{c}=-\omega^{2} M_{p, l u m p} u_{i}+j \omega R_{p, l u m p} u_{i}+\frac{1}{C_{p, l u m p}} u_{i}+j \omega Z_{F E M} u_{i}
$$

As shown in Fig. 1 the coupling node $i$ defines which node in the FE system the LPM should couple to. In order to identify the degree of freedom (DOF) the contribution from the LPM should be added to, a set of indicator matrices are created

$$
\mathbf{I}_{i r}=\mathbf{e}_{i d o f r} \mathbf{e}_{i d o f r}^{T} \quad, \quad \mathbf{I}_{i z}=\mathbf{e}_{i d o f z} \mathbf{e}_{i d o f z}^{T} \quad, \quad \mathbf{L}=\mathbf{e}_{l} \mathbf{e}_{l}^{T},
$$

where $\mathbf{e}_{\text {idofr } r}$ and $\mathbf{e}_{i d o f z}$ are zero vectors except with a unit entry corresponding to the $r$ and $z$ DOF of the coupling node $i$ and $\mathbf{e}_{l}$ is a zero vector with a unit entry in the last component. An indicator matrix, $\mathbf{J}_{i z}$, is also created which has a unit entry in the bottom row corresponding to the $z$ DOF.

The scalar equation (18) is added to Eq. (16) by multiplying the lumped components onto the indicator matrices in Eq. (19). This yields the system of equations for the hybrid FE-LPM model

$$
\begin{array}{r}
\left(\left[\begin{array}{cc}
\tilde{\mathbf{K}} & \mathbf{0} \\
\mathbf{0} & 0
\end{array}\right]+\frac{1}{C_{p, l u m p, r}} \mathbf{I}_{i r}+\frac{1}{C_{p, l u m p, z}} \mathbf{I}_{i z}+R_{E} \mathbf{L}-B l \mathbf{J}_{i z}^{T}\right. \\
+j \omega\left(R_{p, l u m p} \mathbf{I}_{i z}+B l \mathbf{J}_{i z}\right)+(j \omega)^{n} L_{E} \mathbf{L} \\
\left.-\omega^{2}\left(\left[\begin{array}{cc}
\tilde{\mathbf{M}} & \mathbf{0} \\
\mathbf{0} & 0
\end{array}\right]+M_{p, l u m p} \mathbf{I}_{i z}\right)\right)\left\{\begin{array}{l}
\mathbf{u} \\
\mathbf{p} \\
i_{c}
\end{array}\right\}=\left\{\begin{array}{c}
\mathbf{0} \\
\mathbf{0} \\
e_{g}
\end{array}\right\}
\end{array}
$$

where $\mathbf{J}_{i z}^{T}$ is the transpose of $\mathbf{J}_{i z}, C_{p, l u m p, r}$ and $C_{p, \text { lump, } z}$ refers, respectively, to the lumped compliance in the $r$ and $z$ direction. The rest of the lumped parameters are only applied in the z-direction, which is the direction of the applied force. The FE equations in Eq. 20 are solved with an in-house Matlab code. 


\subsection{Estimation of optimal lumped parameters}

Two of the most distinctive parameters used to identify the performance of a given loudspeaker are the pressure 1 meter away from the speaker and the electrical impedance of the VC. These physical quantities are used to formulate an objective function, such that the measured speaker response can be matched with the numerical model. The following objective function considers the absolute error squared, meaning the square of the norm of the complex difference between the numerical model and the measured data, which is normalized with respect to the square of the norm of the measured data, which in this paper comes from a numerical experiment carried out on a full model of a loudspeaker

$$
\phi=\frac{\left\|\mathbf{p}_{\text {meas }}-\mathbf{p}\right\|_{2}^{2}}{\left\|\mathbf{p}_{\text {meas }}\right\|_{2}^{2}}+\frac{\left\|\mathbf{z}_{\text {meas }}-\mathbf{z}\right\|_{2}^{2}}{\left\|\mathbf{z}_{\text {meas }}\right\|_{2}^{2}} .
$$

Here $\phi$ is the value of the objective function, $\mathbf{p}_{\text {meas }}$ is the measured pressure $1 \mathrm{~m}$ away from the speaker at different frequencies, $\mathbf{p}$ is the computed pressure, $\mathbf{z}_{\text {meas }}$ is the measured $\mathrm{VC}$ impedance and $\mathbf{z}$ is the simulated $\mathrm{VC}$ impedance. The optimization problem is stated in Eq. (22), the associated constraints, $L_{l}$ and $L_{h}$ are defined in Tab 1.

$$
\begin{array}{cl}
\min _{\mathbf{x}} & \phi(\mathbf{x}), \\
\text { s.t. } & \text { Eq. }(13), \\
& L_{l} \leq x_{k} \leq L_{h}, \quad k=1, \ldots, 6,
\end{array}
$$

\subsubsection{Adapting the proposed method for an experimental setup}

The proposed method takes into account both the magnitude and phase of both the pressure and the impedance. This is done such that the motion of the speaker in the numerical model can match the motion of a measured speaker. In this work we match our numerical model with a numerical reference model from Comsol. The objective function is, however, constructed in such a way that the data obtained from Comsol in principle could be replaced with measurements. These measurements should be done in an anechoic chamber with a pressure microphone and electrical equipment to measure the impedance. From these measurements one can extract the magnitude and phase from the pressure and impedance measurements, respectively.

If an anechoic chamber is not readily available another physical quantity that describes the motion of the speaker, such as the velocity of the diaphragm, could be used. The measurement of the velocity could be carried out with e.g. the Klippel system that utilizes a system identification technique based on impedance- and velocity measurements.

\section{$2.4 \quad$ Numerical setup of reference examples}

The goal is to match the FE-LPM model with a reference loudspeaker. In this paper it is chosen to carry out a numerical experiment by simulating a full loudspeaker in Comsol Multiphysics. The reference model constitutes of the loudspeaker in Fig. 2 and the system in Fig. 4, here, the loudspeaker diaphragm is attached to the VC former as indicated with the dashed line. The system on Fig. 4 consists of the electric motor system, VC, VC former and spider which is exactly the system in the dashed box on Fig. 1. The VC consists of 100 windings, the wire has a thickness of $0.3 \mathrm{~mm}$ and it is made of copper. The VC-former is made of aluminum.

The reference model is a 2D axisymmetric model, where linear elasticity is assumed, the acoustics are computed without including viscous losses, the pole piece is made of iron with non-linear magnetic material properties. The solution procedure follows that of the example in Ref. [24]. First, the problem is considered static and solved inorder to extract the driving force factor and local permeability. The subsequent full analysis considers the stationary response of the loudspeaker and utilizes the former stationary solution as a linearization point. This numerical analysis includes fully coupled physics, which means that Lorentz coupling is used to describe the electromagnetic force acting on the moving VC in a magnetic field and acoustic-structure interaction is used to model the sound propagation from the moving loudspeaker.

The reference model is a full model including the entire magnet system which means that this model consists of 584072 DOF. This is computationally heavier compared to our proposed FE-LP method which has 217309 DOF. The discrepancy in complexity between the two models can mainly be attributed to the entire magnet system that 


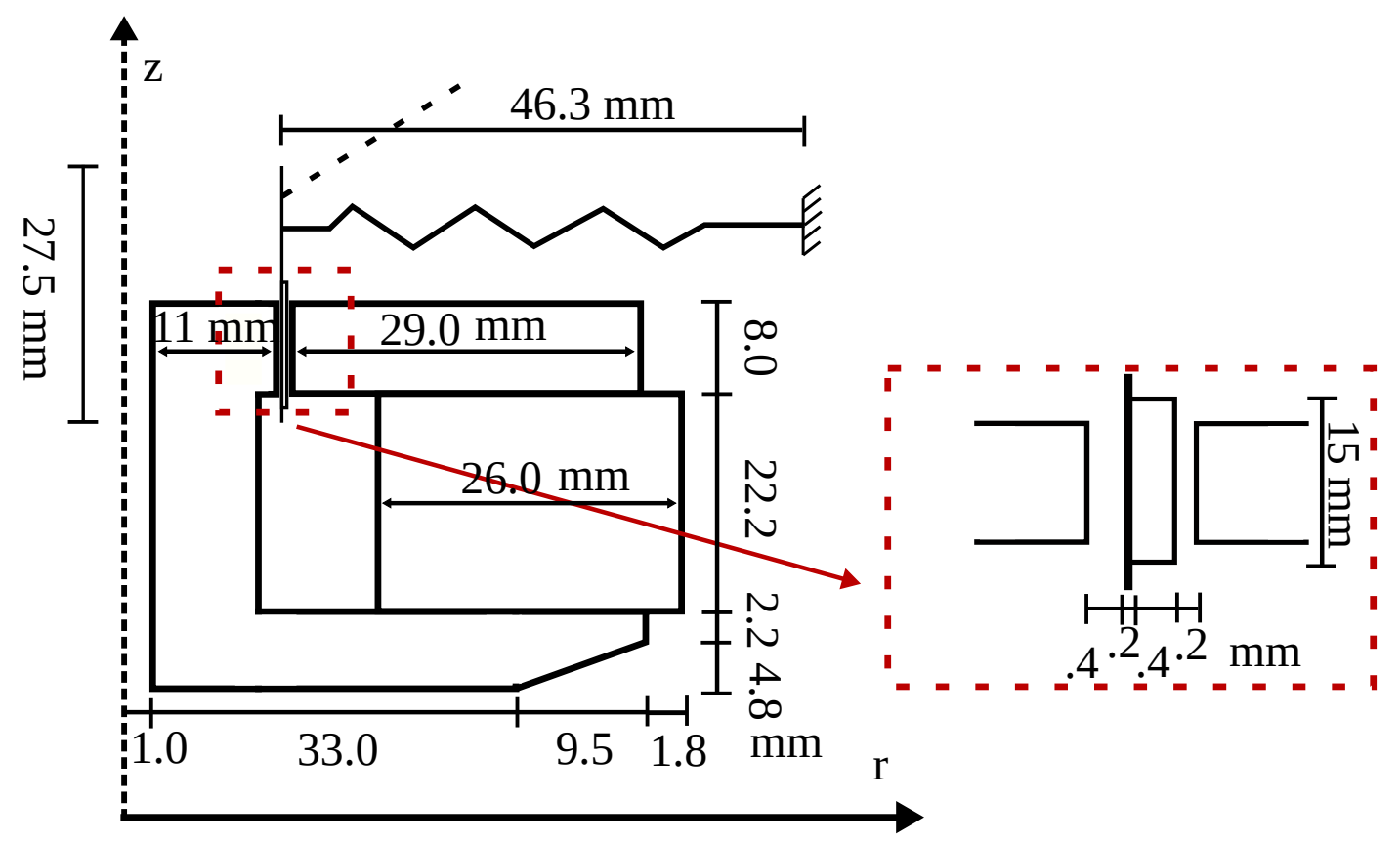

Figure 4: Sketch of the electric motor system, VC and VC-former which is included in the FE reference model.

Table 1: Table of values used as a starting guess for the optimization together with the constraint of each individual variable

\begin{tabular}{l|c|c|c|c|c|c}
$x_{k}$ & $L_{E}[\mathrm{mH}]$ & $n[-]$ & $C_{p, \text { lump }, z}[\mathrm{~m} / \mathrm{N}]$ & $C_{p, \text { lump }, r}[\mathrm{~m} / \mathrm{N}]$ & $R_{p, \text { lump }}[\mathrm{Ns} / \mathrm{m}]$ & $B l[\mathrm{Tm}]$ \\
\hline Start & 2.0 & 0.7 & $4 \cdot 10^{-3}$ & $4 \cdot 10^{-6}$ & 1.2 & 4.0 \\
{$\left[L_{l} ; L_{h}\right]$} & {$[0.1 ; 6.0]$} & {$[0.2 ; 0.9]$} & $4 \cdot\left[10^{-6} ; 10^{-1}\right]$ & $4 \cdot\left[10^{-9} ; 10^{-4}\right]$ & {$[0.24 ; 6]$} & {$[3.5 ; 7.5]$}
\end{tabular}

needs to be meshed. The mesh for the reference model contains many small details such as the gap between the $\mathrm{VC}$ and the pole piece or the very thin $\mathrm{VC}$ former.

\section{Results}

The target for the optimization is to find a set of suitable values for the lumped parameter elements in Eq. (20). An initial guess of the value of the lumped parameters is required, these values are shown together with the constraints used for each variable during the optimization in Tab. 1.

Two of the lumped parameters are not included in the optimization, one of them is the DC resistance, $R_{E}$, of the $\mathrm{VC}$ which can be found from the impedance measurement. The value of $R_{E}$ is $3.54 \Omega$. The second is $M_{p, l u m p}$ which can be found by weighing the spider and VC components, $M_{p, l u m p}$ has a value of $0.006 \mathrm{~kg}$. Due to the fact that a numerical experiment is used we know the force factor, however, this would not be the case for an experimental set-up of a authentic loudspeaker. Therefore we consider $B l$ as unknown and as a consequence of that it is included in the optimization. The input into the electrical motor system is an $\mathrm{AC}$ voltage source with the value of $1 \mathrm{~V}$.

We apply sequential quadratic programming (SQP) to solve the optimization problem in Eq. (22). The SQP algorithm is used by calling the internal Matlab function fmincon. The gradients used in the optimization is computed with finite difference using the default step length $\sqrt{\epsilon}$. Using the SQP algorithm to minimize Eq. (21), on the system of equations in Eq. (20), using the starting guess in Tab. 1 yields the design history in Fig. 5.

The values of the LPM that minimizes the objective function the most are presented in Tab. 2. We note that the value found for the force factor is $4.83 \mathrm{~T} \cdot \mathrm{m}$ which is only $3 \%$ deviation from the calculated value of $4.67 \mathrm{~T} \cdot \mathrm{m}$. 


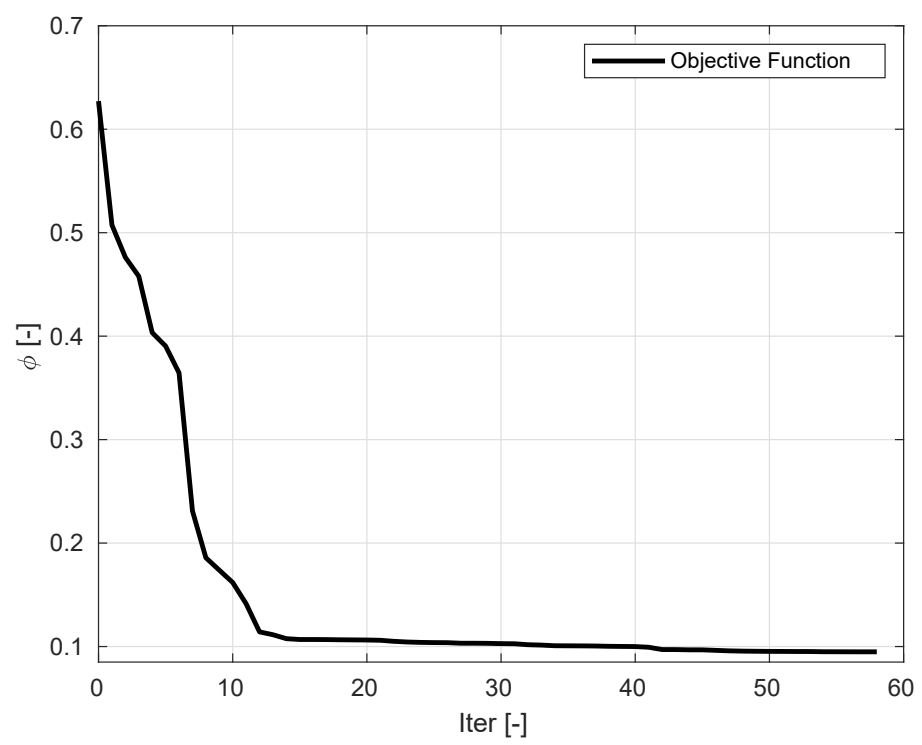

Figure 5: The history of the optimization, where the y-axis represents the value of $\phi$ for each iteration step.

Table 2: Table containing the optimally estimated lumped parameters

\begin{tabular}{c|c|c|c|c|c}
$L_{E}[\mathrm{mH}]$ & $n[-]$ & $C_{p, \text { lump }, z}[\mathrm{~m} / \mathrm{N}]$ & $C_{p, \text { lump }, r}[\mathrm{~m} / \mathrm{N}]$ & $R_{p, \text { lump }}[\mathrm{N} \cdot \mathrm{s} / \mathrm{m}]$ & $B l[\mathrm{~T} \cdot \mathrm{m}]$ \\
\hline 3.63 & 0.77 & $4.81 \cdot 10^{-4}$ & $4 \cdot 10^{-9}$ & 0.53 & 4.83
\end{tabular}

We can also observe that the compliance in the $r$ direction reaches its lower bound, if this bound is lowered even further the compliance will also reach that. This finding makes sense since the compliance in the $r$ direction keeps the loudspeaker centred, ideally, the stiffness in this direction would be infinite.

The optimal values of Tab. 2 are analysed by computing the pressure response 1 meter away from the speaker as a function of frequency. These results are compared with results for a full loudspeaker model and results for the values of the starting guess in Tab. 1. This is shown in Fig. 6 .

From Fig. 6 one can observe that the optimization algorithm is able to tune the lumped parameters such that the the FE-LPM model matches the numerical experiment. A discrepancy between the two models at $316 \mathrm{~Hz}$ can be observed. The discrepancy can be attributed to the lack of viscous and thermal losses in the reference model in the small slits between the VC and the pole piece and the tube near the z-axis, these features are shown in Fig. 4. It is expected that viscous and thermal losses can have an effect on the actual response, which is to reduce the cavity resonance effect compared to the lossless counterpart. The cavity resonance at $316 \mathrm{~Hz}$ manifests itself in both the pressure and impedance response. In practice this resonance will hardly be present, this is mainly due to losses being present but also due to air-vents in the $\mathrm{VC}$ former, which prevents a pressure build-up. The discrepancy is mainly due to model simplifications in the reference loudspeaker and are therefore not considered important.

The values of Tab. 2 are used to simulate the impedance of the $\mathrm{VC}$ as a function of frequency. Again, the obtained response is compared with the reference values from the numerical experiment and the initial guess. This comparison can be seen in Fig. 7.

Figure 7 shows that the FE-LPM and the numerical experiment has a good agreement from $1 \mathrm{~Hz}$ to approximately $4 \mathrm{kHz}$, above $4 \mathrm{kHz}$ the FE-LPM model starts to deviate from the reference as the frequency increases. No immediate explanation exist for this deviation, however, some deviation is to be expected due to high-frequency effects that are present in the full model but not in our simplified approach with lumped mechanical and electrical components. The full model includes the spider and VC former, where break-up modes will be present at high frequencies, besides this difference the two FE models have identical mechanical components. 


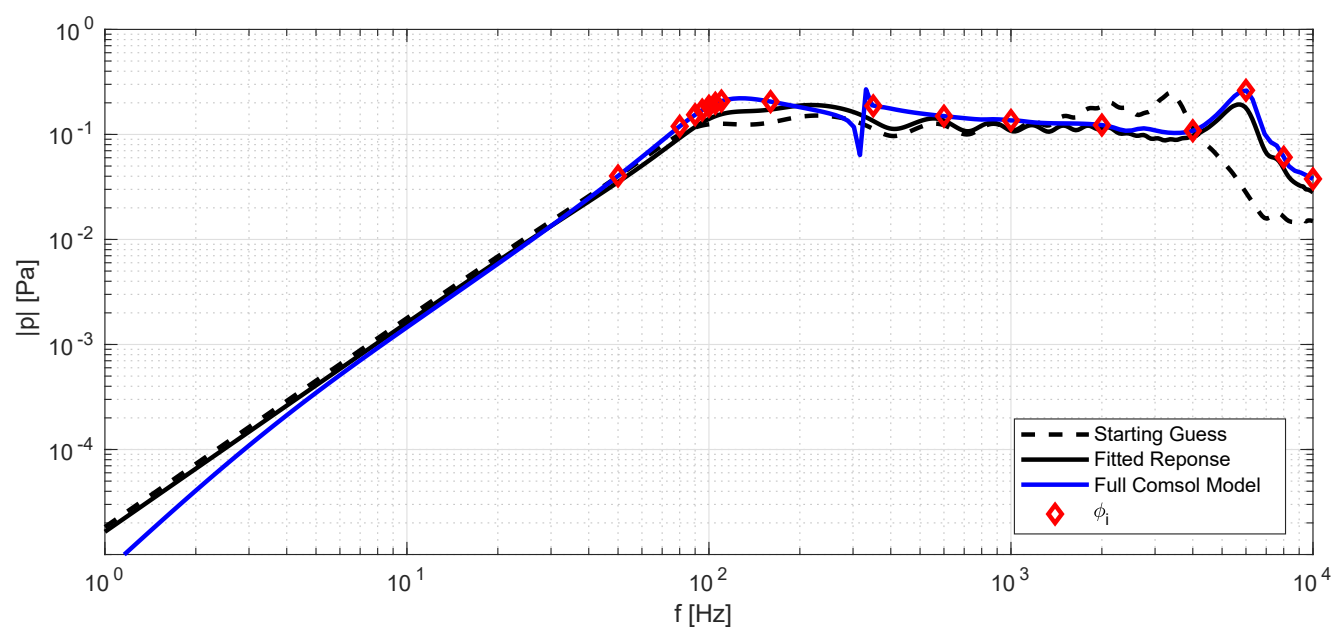

Figure 6: Pressure response between $1 \mathrm{~Hz}$ and $10 \mathrm{kHz}$. The solid black line is the result of the optimization, the solid blue line is the full loudspeaker model, the dashed black line is the starting guess and the red diamond-shaped discrete points are the frequencies which are used in the optimization.

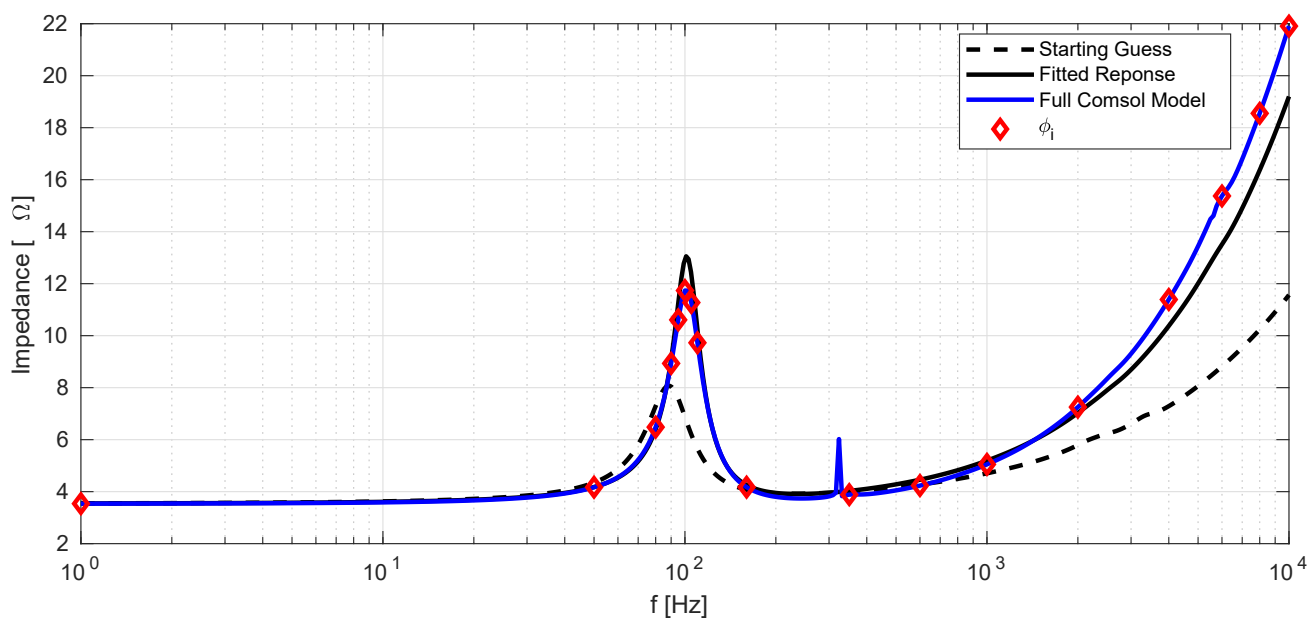

Figure 7: Impedance between $1 \mathrm{~Hz}$ and $10 \mathrm{kHz}$. The solid black line is the result of the optimization, the solid blue line is the full loudspeaker model, the dashed black line is the starting guess and the red diamond-shaped discrete points are the frequencies which are used in the optimization. 


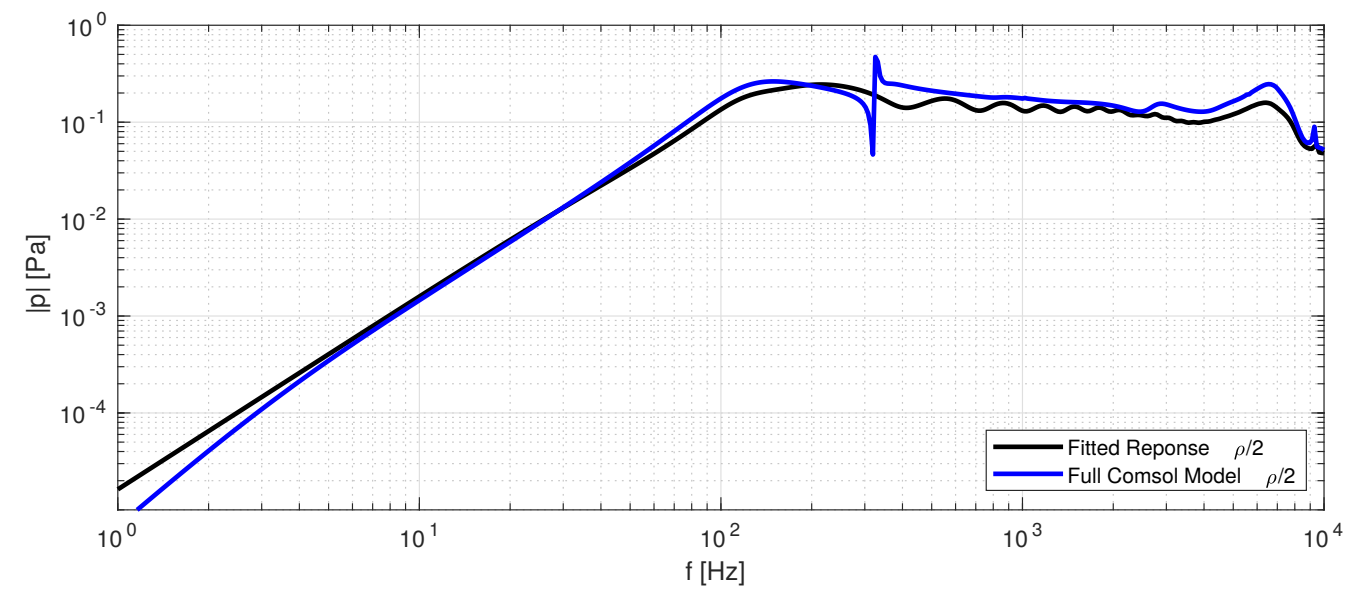

Figure 8: Pressure response between $1 \mathrm{~Hz}$ and $10 \mathrm{kHz}$. The solid black line is the result of the optimization, the solid blue line is the full loudspeaker model.

\subsection{Robustness of the method}

This section presents a test case which demonstrates the robustness of the proposed method. It is necessary to test whether the results obtained in the above section rely on a very specific tuning of the lumped parameters. Ideally changes to the baseline configuration of the reference loudspeaker and the FE-LPM should not cause a deviation between the two models. However, if the above results are a product of a fortunate tuning of the lumped parameters that only works in this specific case, the results will be very sensitive to changes in the baseline configuration. That means that one should observe a major deviation between the output of the reference loudspeaker compared to the proposed FE-LPM. The change in the models will be a change of the density of the loudspeaker diaphragm and dust cap. The density will be halved in these structural regions in both the reference loudspeaker and in the FE-LPM model. The rest of the material parameters in the FE-model will remain unchanged as will the values in the LP-model.

Figure 8 shows the pressure response of the test case. It is observed that the trends from Fig. 6 is preserved and an overall satisfactory agreements is reached between the two models. One can observe a spike in the pressure at $9.2 \mathrm{kHz}$ which is attributed to a breakup mode that is now present due to the lower density of the diaphragm.

Figure 9 shows the impedance as a function of frequency. Again, the trends from Fig. 7 is also present for the test case. Figure 9 demonstrates that the FE-LPM accurately captures that the first eigenfrequency of the structure is slightly higher than that of Fig. 9. This shift of the natural resonance frequency is to be expected since the mass of the diaphragm and dust cap is lower.

Generally the test case shows that the FE-LPM adapts well to changes. When comparing the FE-LPM model with the reference loudspeaker we see the same trends as in the previous section. We note the the FE-LPM captures the break-up mode at high frequencies and the shift in the fundamental resonance frequency of the loudspeaker.

\section{Discussion}

This paper presents a method that can be utilized together with structural optimization. The method lumps certain parts of the loudspeaker to create a computational speedup. However, this method is not limited to the model problem in this paper. The presented method can be extended, such that it can be applied on different model problems.

In this paper we find values for the lumped components, that works well in the frequency range from $1 \mathrm{~Hz}$ to $10 \mathrm{kHz}$, in reality the components are frequency dependent. Therefore, the presented model could be used to solve several optimization problems at different frequencies and thus obtaining a frequency dependent mechanical stiffness 


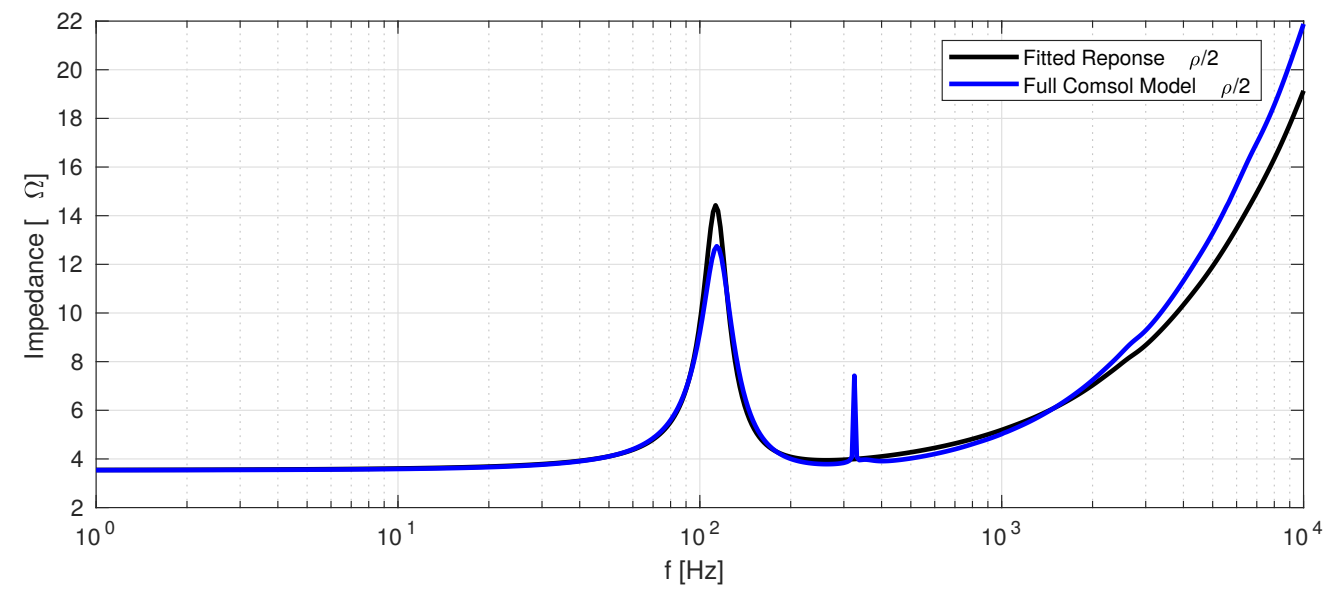

Figure 9: Impedance between $1 \mathrm{~Hz}$ and $10 \mathrm{kHz}$. The solid black line is the result of the optimization, the solid blue line is the full loudspeaker model.

and damping. Taking this approach one step further would include lumping the surround as well and establishing a fractional order derivative model of the surround and by that account for the viscoelastic effects in the rubber.

One could consider lumping only the electrical system and therefore include the spider, VC and VC former in the FE model. This approach could be used to optimize the electrical components of a loudspeaker.

Currently 17 discrete points are used to evaluate the objective function, as shown in Fig. 6 and 7 . The amount of points are limited to 17 due to the computational effort required to carry out the optimization. This can be attributed to the fact that finite difference is used to calculate the gradients used in the optimization. A more efficient way would be to compute the gradients with the adjoint approach. Another approach than the one presented in this paper would be to compute the mechanical impedance of the FE model together with a transfer function related to the pressure 1 meter away from the speaker, these quantities would replace the FE model. This will greatly reduce the DOF in the model, which will allow for more discrete points in the optimization. This will be a very relevant approach if the end goal is only to consider lumped parameter estimation.

The objective function in Eq. (21) considers the pressure generated by the loudspeaker and the impedance of the VC. However, if there is no reliable way of obtaining the pressure one could possibly use a laser to measure the velocity of the diaphragm and use that quantity in the objective function.

\section{Conclusion}

This paper presents a methodology for improving the computational efficiency of FE models of loudspeaker while preserving the accuracy of the model for a specific purpose. This is illustrated by lumping the motor system and parts of the mechanical components. We demonstrate that the approach is able to closely mimic the pressure and impedance response of a full loudspeaker model even at high frequencies. The presented method can be used together with structural optimization of the diaphragm, dust cap and surround. Simple model effects such as mechanical damping and electrical inductance were used but more sophisticated models are straightforward to include.

\section{References}

[1] J. Kook and J. S. Jensen, Topology optimization of periodic microstructures for enhanced loss factor using acoustic-structure interaction, International Journal of Solids and Structures 122-123 (2017) 59-68.

[2] L. Shu, M. Yu Wang and Z. Ma, Level set based topology optimization of vibrating structures for coupled acoustic-structural dynamics, Computers and Structures 132 (2014) 34-42. 
[3] S. Marburg, Developments in structural-acoustic optimization for passive noise control, Archives of Computational Methods in Engineering 9 (2002) 291-370.

[4] G. H. Yoon, J. S. Jensen and O. Sigmund, Topology optimization of acoustic-structure interaction problems using a mixed finite element formulation, International Journal for Numerical Methods in Engineering $\mathbf{7 0}$ (2007) 1049-1075.

[5] M. P. Bendsøe and O. Sigmund, Topology optimization Theory, Methods and Applications (Springer, Berlin, Heidelberg, New York, 2004), 2 edition.

[6] A. Bezzola, Numerical optimization strategies for acoustic elements in loudspeaker design, 145th Audio Engineering Society International Convention, Aes 2018.

[7] P. Macey and K. Griffiths, Automated design of loudspeaker diaphragm profile by optimising the simulated radiated sound field with experimental validation, 128th Audio Engineering Society Convention 20103 (2010) $1777-1788$.

[8] R. D. Cook, D. S. Malkus, M. E. Plesha and R. J. Witt, Concepts and Applications of Finite Element Analysis (John Wiley \& Sons, 2002), 4 edition.

[9] W. M. Leach Jr., Introduction to Electroacoustics \&3 Audio Amplifier Design (Kendal/Hunt Publishing Company, 2003), 3rd edition.

[10] W. Sun and W. Hu, Integrated fe-le modelling method for a simplified balanced-armature receiver, International Journal of Acoustics and Vibrations 22 (2017) 377-383.

[11] G. Schrag, T. Kunzig and A. Dehe, Enhanced design of microsystems by combining lumped and distributed system-level models, Symposium on Design, Test, Integration and Packaging of Mems/moems, Dtip 2016 (2016) 7514839.

[12] Comsol Multiphysics, Lumped receiver with full vibroacoustic coupling, https://www.comsol.com/model/ lumped-receiver-with-full-vibroacoustic-coupling-61751, accesed 27-1-2020.

[13] P. Marttila and M. J. H. Jensen, A hybrid electroacoustic lumped and finite element model for modeling loudspeaker drivers, in Audio Engineering Society Conference: 51st International Conference: Loudspeakers and Headphones (2013).

[14] Klippel Gmbh, Specification of the klippel analyzer system, www.klippel.de, accesed 12-02-2020.

[15] W. Klippel and J. Schlechter, Distributed mechanical parameters of loudspeakers part 1: Measurements, Journal of the Audio Engineering Society 57 (2009) 500-511.

[16] W. Cardenas and W. Klippel, Optimal material parameter estimation by fitting finite element simulations to loudspeaker measurements, 144th Audio Engineering Society Convention 2018.

[17] D. G. Nielsen, J. S. Jensen, V. Cutanda Henriquez and F. T. Agerkvist, Finite element model coupled with lumped parameter elements, Proceedings of 14 th International Conference on Theoretical and Computational Acoustics (2019) 297-304.

[18] J. P. Bérenger, Perfectly matched layer for the fdtd solution of wave-structure interaction problems, Ieee Transactions on Antennas and Propagation 44 (1996) 110-117.

[19] J. N. Li, S. X. Wang, H. J. Yin, C. H. Dong and H. M. Chen, Acoustic wave equation modeling in cylindrical coordinates with convolutional pml, 77th Eage Conference and Exhibition 2015: Earth Science for Energy and Environment (2015) 4546-4548.

[20] F. Collino and P. Monk, The perfectly matched layer in curvilinear coordinates, Siam Journal of Scientific Computing 19 (1998) 2061-2090. 
[21] J. S. Jensen, Topology optimization problems for reflection and dissipation of elastic waves, Journal of Sound and Vibration 301 (2007) 319-340.

[22] A. W. King and F. T. Agerkvist, Fractional derivative loudspeaker models for nonlinear suspensions and voice coils, Journal of Audio Engineering Society 66 (2018) 525-536.

[23] P. Brunet and B. Shafai, Identification of loudspeakers using fractional derivatives, Aes: Journal of the Audio Engineering Society 62 (2014) 505-515.

[24] Comsol Multiphysics, Loudspeaker driver - frequency-domain analysis, https://www.comsol.com/model/ loudspeaker-driver-8212-frequency-domain-analysis-1369, accesed 27-1-2020. 